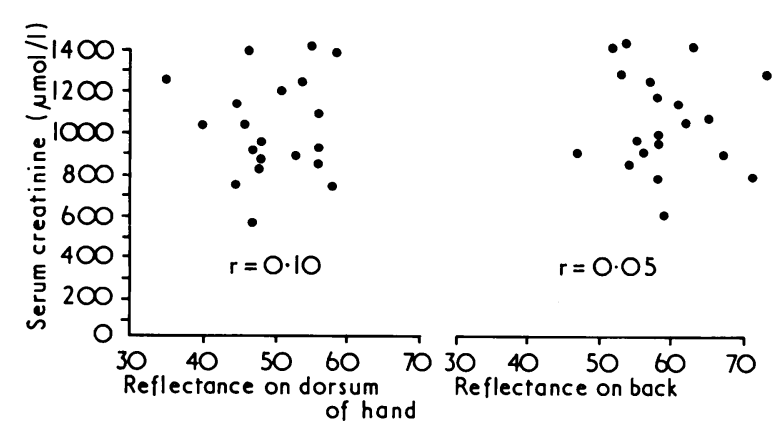

FIG. 4-Lack of correlation between skin pigmentation as measured by reflectance photometry and renal function as measured by serum creatinine in 20 patients.

serum creatinine, and degree of pigmentation may have been partly due to the effect of dialysis, but more probably it was due to the severity of the renal failure, and we are now looking for a relationship in patients with less impairment of renal function.

Our findings may explain the pigmentation of chronic renal failure. Certainly the levels of immunoreactive $\beta-\mathrm{MSH}$ we found are similar to those which occur in Addison's disease and Nelson's syndrome. ${ }^{2-4}$ It has been suggested, however, that $\beta-$ MSH in man exists only as part of the larger lipotrophin (LPH) molecule. ${ }^{5}$ If this is true then the $\beta-M S H$ we have measured may have been the immunoreactive moiety contained within the parent LPH molecule, and the concentration of LPH may therefore be increased in chronic renal failure. But there are problems in attributing the pigmentation of chronic failure to LPH because this hormone is thought to have only $0.6 \%$ of the pigmentary potency of $\beta$-MSH. ${ }^{\circ}$

Several possibilities may explain this discrepancy. Firstly, the pigmentary potency of LPH in the human melanocyte may be greater than its potency in the amphibian melanophore, and it may be wrong to equate mammalian and amphibian pigmentary responses. Secondly, LPH may be a prohormone and an increase in its plasma concentration may produce an increase in the active hormone (? $\beta-\mathrm{MSH}$ ) at the target organ, the melanocyte. Thus though we may have been measuring increases in LPH in plasma the pigmentation may still be due to $\beta-M S H$ formed at the target tissue itself. Thirdly, if $\beta-\mathrm{MSH}$ is normally split off from LPH in vivo (not just at the target organ) it would be cleared by the kidney so that little would be detected in the plasma. In chronic renal failure, by contrast, $\beta-\mathrm{MSH}$ would not be cleared and its accumulation would explain the pigmentation. Thus we may have been measuring a mixture of $\beta-M S H$ and LPH in the plasma. Lastly, MSH peptides other than $\beta-M S H$ and LPH may occur in man.

As we are uncertain about the relation between the concentration of active hormone at the melanocyte target and plasma concentration of MSH peptides it is perhaps not surprising that we found no correlation between plasma immunoreactive $\beta-\mathrm{MSH}$ and the degree of skin pigmentation. Also, much of the colour may be due to pigments other than melanin. ${ }^{78}$ Thus while the increase in immunoreactive $\beta-\mathrm{MSH}$ we found in chronic renal failure could well be a factor in the pigmentation the precise relation of the MSH peptides to the pigmentation must await resolution of all these difficulties.

A.G.S. gratefully acknowledges a Fellowship in dermatology from the Wellcome Trust. F.A-U. is supported by the Fundacion J. March of Spain. We also wish to acknowledge a grant from the Medical Research Council.

\section{References}

1 Comaish, J. S., Ashcroft, T., and Kerr, D. N. S., Acta Dermato-venereologica, 1975. In press.

2 Thody, A. J., and Plummer, N. A., Fournal of Endocrinology, 1973, 58, 263. 3 Abe, K., et al., Fournal of Clinical Investigation, 1967, 46, 1609.

4 Abe, K., et al., Fournal of Clinical Investigation, 1968, 48, 1580.

5 Bloomfield, G. A., et al., Nature, 1974, 252, 492.

6 Chretien, M., Methods in Investigative and Diagnostic Endocrinology, 1973, 2, 617 .

7 Tsaltas, T. T., Transactions. American Society for Artificial Internal Organs, 1969, 15, 321.

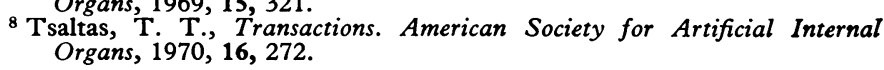

\title{
New Serological Test for Malaria Antibodies
}

\author{
A. VOLLER, G. HULDT, C. THORS, E. ENGVALL
}

British Medical fournal, 1975, 1, 659-661

\section{Summary}

In an enzyme-linked immunosorbent assay test for malaria antibodies, antibodies to Plasmodium vivax and $P$. falciparum in man are detected using a crude antigen prepared from the simian malaria parasite $P$. knowlesi. The test may be suitable for epidemiological studies.

\footnotetext{
London School of Hygiene and Tropical Medicine, London N.W.1 A. VOLLER, D.sc., Senior Lecturer, Department of Clinical Tropical Medicine

National Bacteriological Laboratory, Stockholm G. HULDT, M.D., Chief, Parasitology Section C. THORS, B.SC., Technical Assistant

University of Stockholm

E. ENGVALL, B.Sc., Research Assistant
}

\section{Introduction}

The value of serological studies in supplementing parasitological data in the epidemiological assessment of malaria is generally recognized. Various serological techniques, especially immunofluorescence and passive haemagglutination and gel diffusion tests, have been used but none are entirely satisfactory. There is still a place for a test which may be used on a large scale and in which the results are read objectively. The enzyme-linked immunosorbent assay (E.L.I.S.A.) technique developed by Engvall and Perlmann ${ }^{12}$ and Engvall et al. ${ }^{3}$ for measuring antigens and antibodies has been used successfully to measure antibodies to Trichinella spiralis. ${ }^{4-6} \mathrm{We}$ therefore decided to see whether it could be applied as a test for the presence of malaria antibodies.

PRINCIPLE OF TEST (fig. 1)

A soluble antigen is immobilized by coating a solid base such as a plastic tube or plate. The test serum thought to contain antibody to the antigen is incubated in the coated tube. Any specific antibody present will react with the antigen. The tube is then washed to remove 
all free immunoglobulin. A solution of conjugate consisting of antispecies globulin labelled with enzyme is then incubated in the tube. This reacts with the antibody fixed to the antigen. Further washing removes excess conjugate. The enzyme substrate is then added and its change of colour indicates the amount of enzyme in the tube. This amount is proportional to the concentration of antibody in the test serum.

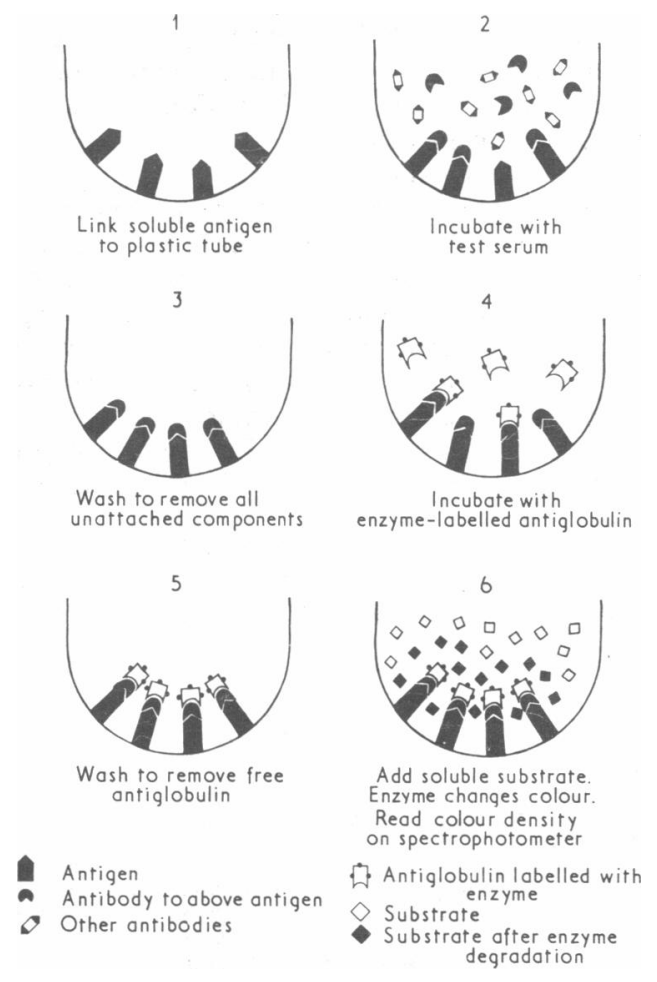

FIG. 1-Principle of E.L.I.S.A. test.

\section{Materials and Methods}

SERA

In a first trial the sera tested were from $(a)$ a patient who had had a recent Plasmodium falciparum infection and had an immunofluorescent IgG titre of $1 / 2560 ;(b)$ a Swedish blood donor who had never been in the tropics; $(c)$ samples from 20 patients in the Pare area of Tanzania, of whom 10 had falciparum parasitaemia and 10 were parasitologically negative (the incidence of malaria is high in this area and probably these persons would have been exposed to infection); and (d) samples from 20 Swedish blood donors who had not visited the tropics.

In a second trial the sera tested were from $(a)$ and $(b)$ above and $(e)$ samples from 96 patients in the Bandar Abbass area of Southern Iran, 23 of whom had falciparum infections, 18 vivax infections, and 55 no demonstrable parasites. Malaria is endemic in this area but transmission is much less than in Pare.

\section{ANTIGEN}

The antigen used was derived from $P$. knowlesi. This virulent simian parasite was inoculated into the blood of Macaca mulatta monkeys and the course of the infection monitored by daily blood film examination. The monkeys were killed when the parasitaemia exceeded $20 \%$ and the parasites were in the mature schizont stage. The schizontinfected erythrocytes in heparinized blood samples were concentrated into an upper brown layer by centrifugation at $1000 \mathrm{~g}$ for 15 minutes. The leucocyte layer above the schizont-infected erythrocytes was discarded, the brown-layer material was washed three times in

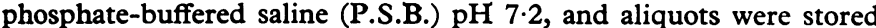
as pellets at $-70^{\circ} \mathrm{C}$. Soluble antigen was prepared by sonic disruption of the schizonts. ${ }^{7}$ The schizont pellet was suspended in nine times its volume of P.B.S. pH 7.2 and subjected to 20 seconds of ultrasonics at force 4 on a Bronson sonifier. Then the suspension was centrifuged at $4^{\circ} \mathrm{C}$ at $8500 \mathrm{~g}$ for 15 minutes. The supernatant (the soluble antigen) was stored at $-70^{\circ} \mathrm{C}$ until used for coating the tubes.

\section{PERFORMANCE OF TEST}

The methods of Engvall and Perlmann ${ }^{2}$ were followed.

(1) The soluble malaria antigen solution was diluted with $0.05 \mathrm{M}$ carbonate buffer $\mathrm{pH} 9.6$ containing $0.02 \%$ sodium azide to $5 \mu \mathrm{g}$ protein $/ \mathrm{ml} ; 1 \mathrm{ml}$ of this solution was added to each plastic (polystyrene) tube. The tubes were incubated upright at room temperature overnight and then stored at $4^{\circ} \mathrm{C}$ until needed. Before use the tubes were washed twice with saline containing $0.05 \%$ polysorbate 20 .

(2) The test serum was diluted $1 / 1000$ in P.B.S. $\mathrm{pH} 7 \cdot 2$ containing $0.05 \%$ polysorbate 80 and $0.02 \%$ sodium azide, $1 \mathrm{ml}$ being added to each tube. Control tubes containing only P.B.S. were also set up. The tubes were incubated for two hours on a roller drum at room temperature.

(3) The tubes were washed three times with saline-polysorbate 20 .

(4) The alkaline-phosphatase-labelled antihuman globulin conjugate was diluted to a suitable concentration (1/800 in this study) determined by testing it in tubes coated with a reference $\mathrm{IgG}$ preparation; $1 \mathrm{ml}$ was added to each tube and the tubes were incubated for three hours at room temperature.

(5) The tubes were washed three times in saline-polysorbate 20

(6) Diethanolamine buffer $(\mathrm{pH} 9.8) 1 \mathrm{ml}$ containing $1 \mathrm{mg} p$ nitrophenylphosphate and $0.5 \mathrm{mmol} \mathrm{MgCl} / 1$ was added to each tube. When a yellow colour developed the enzyme reaction was stopped by adding $0.1 \mathrm{ml} 2 \mathrm{M} \mathrm{NaOH}$. The extinction value at $400 \mathrm{~nm}$ was then measured in a spectrophotometer.

The results of the first trial are expressed as the rate of enzyme activity per 100 minutes (E400/100 minutes). This is calculated as

$$
\mathrm{E} 400 / 100 \text { minutes }=\mathrm{E} 400 \mathrm{~T} \text { minutes } \times \frac{100}{\mathrm{~T}}
$$

where $\mathrm{E} 400$ = optical density or extinction value at $400 \mathrm{~nm}$, and $\mathrm{T}=$ time between adding the substrate and adding the $\mathrm{NaOH}$ to stop the reaction.

The results of the second trial are expressed as E400/60 minutes. In this trial the reaction was stopped 60 minutes after adding the substrate.

\section{Results}

In the first trial the human antiserum to $P$. falciparum (fig. $2(a)$ ) produced a strong reaction with the $P$. knowlesi-antigen-coated tubes. In contrast the serum from the one Swedish blood donor who had never been in the tropics (fig. $2(b)$ ) gave a weak reaction. Absorption of both sera with sheep red blood cells in an attempt to remove nonspecific antibodies did not change the relative reaction values. All but one of the 20 Tanzanian samples (fig. $2(c)$ ) reacted more strongly than the samples from the Swedish blood donor controls (fig. $2(d)$ )

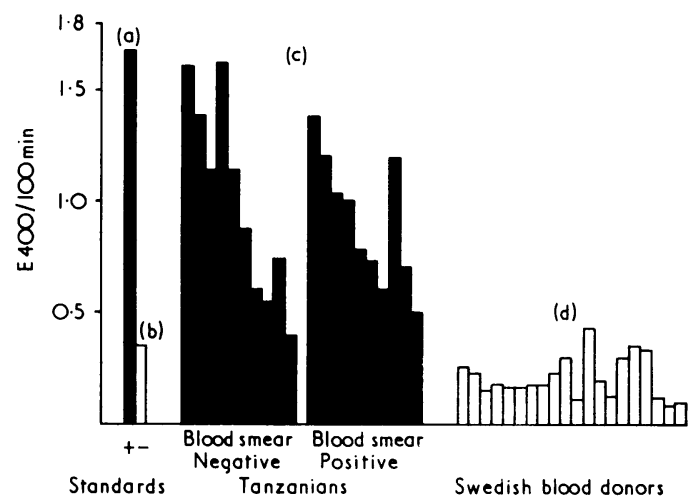

FIG. 2-Results of first trial with sera tested against $P$. knowlesi antigen.

(the one exception was also negative for immunofluorescence). All 10 samples from the Tanzanians with parasitaemia reacted more strongly than those from the controls. There was no difference in mean values between the Tanzanians with parasitaemia and those without.

In the second trial most of the 41 samples from Iranians (fig. 3) 
infected with either $P$. falciparum or $P$. vivax reacted more strongly than those from the controls. The reaction of a few of the 55 parasitologically negative Iranian samples was also stronger than that of the controls. The mean value for the parasitologically negative Iranian samples was below that for the malaria-positive ones.

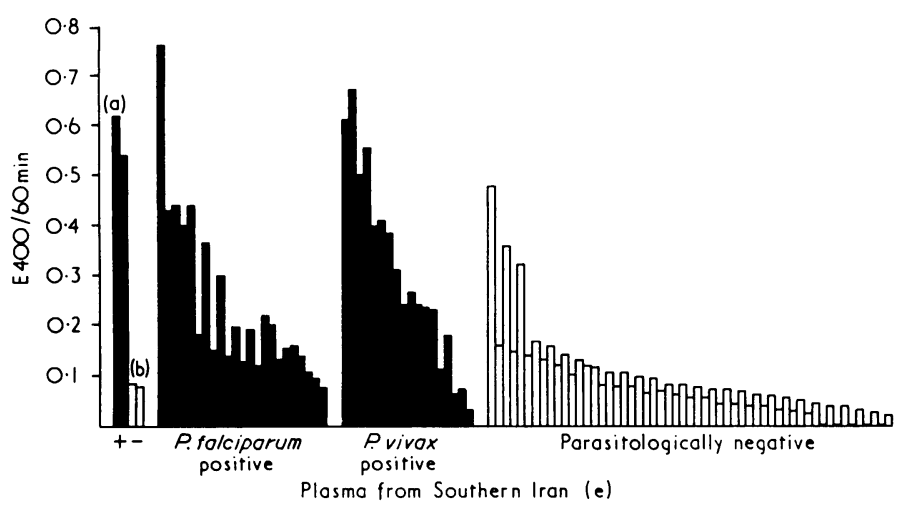

FIG. 3-Results of second trial with sera tested against $P$. knowlesi antigen.

\section{Discussion}

These results show that the enzyme-linked immunosorbent assay detects and measures antibodies to malarial infections. Even when the antigen is derived from $P$. knowlesi, as in this study, the test seems to be sensitive in detecting antibodies to $P$. falciparum and $P$. vivax. When simian parasites have been used to provide the antigen for other malaria serological tests they have generally proved to be less sensitive than those derived from the human parasites. We used P. knowlesi antigen, however, because the large quantities of heavily parasitized blood needed for the development of the test could be obtained from infected Macaca mulatta monkeys, which are readily available. Soon we hope to test antigens prepared from $P$. falciparum and $P$. vivax The antigen used here was a crude extract, and a purified antigen may be expected to have higher specific activity.

Our samples from malaria-infected patients and malariaendemic areas reacted much more strongly than those from the European controls. Almost certainly the reaction is a malariaspecific antigen-antibody one, but we realize the dangers of possible false-positive reactions due to heterophiles, antiglobulins, and "autoimmune" antibodies commonly found in the sera of people living in the tropics. The fact that absorption of the sera with sheep erythrocytes did not reduce their reactivity suggests that heterophiles were not playing an important part. Only extensive tests on many control antisera can rule out all non-specific factors.

The fact that the test gave a positive result in all except one of the Tanzanian samples and yet only half showed parasitaemia shows that it can complement parasitological data by indicating recent as well as current infections.

The tests on the Iranian samples show that good results may be obtained when a time is set for the final enzyme reaction. Used in this way the test is more suitable for routine application. The results in the Iranian samples also show that $P$. vivax is as readily detected as $P$. falciparum. The preponderance of serological negatives among the parasitologically negative samples contrasts with the findings in the Tanzanian samples, virtually all of which were positive. This reflects the much lower malaria endemicity in Iran.

The tests can be carried out on blood samples collected by finger-prick. This and the fact that the samples tested are highly diluted means that the procedure is suitable for large-scale epidemiological programmes using tubes coated with different antigens. Ruitenberg et al. ${ }^{6}$ reported that, fully automated, several-thousand tests can be done daily. Our studies, however, were done with simple, inexpensive equipment well within the reach of most laboratories.

Preliminary trials with the wells of microhaemagglutination plates as the antigen-carrying surface gave promising results. These were read visually. This may lead to a simple field test yielding positive or negative results of possible value for screening.

We thank Dr. G. Heden for his constant encouragement, Dr. G. Edrissian for the Iranian samples, and Mr. Y. G. Matola for the Tanzanian ones. Financial help from the Salen Foundation and the World Health Organization made this work possible.

\section{References}

1 Engvall, E., and Perlmann, P., Immunochemistry, 1971, 8, 871.

2 Engvall, E., and Perlmann, P., fournal of Immunology, 1972, 109, 129. ${ }^{3}$ Engvall, E., Jonsson, K., and Parlmann, P., Biochimica et Biophysica Acta, $1971,251,427$

4 Engvall, E., and Ljungstrom, I., Acta Pathologica et Microbiologica Scandinavica. In press.

${ }^{5}$ Ljungstrom, I., Engvall, E., and Ruitenberg, E. J., in Proceedings of $3 \mathrm{rd}$ International Congress on Parasitology (Munich), vol. 3, p. 1204. 1974. ${ }^{6}$ Ruitenberg, E. J., et al., in Proceedings of 3rd International Congress on

Parasitology (Munich), vol. 3, p. 1203. Vienna, Egerman, 1974.
Meuwissen, J. H. E. T., Leeuwenberg, A. D. E. M., and Molenkamp, G. E., Bulletin of the World Health Organization, 1972, 46, 771.

\section{MEDICAL MEMORANDA}

\section{Cardiac Arrhythmia and Imipramine Therapy}

\section{K. B. RAMANATHAN, C. DAVIDSON}

British Medical fournal, 1975, 1, 661-662

Cardiac arrhythmias are a common problem in patients who attempt suicide with tricyclic antidepressants, but it is less well recognized that arrhythmias may occur with these drugs in the standard therapeutic range. We report here a patient who de-

Cardiac Department, Leeds General Infirmary, Leeds LS1 3EX K. B. RAMANATHAN, M.D., M.R.C.P., Registrar

C. DAVIDSON, M.B., M.R.C.P., Senior Registrar veloped recurrent supraventricular arrhythmias and heart failure during treatment with imipramine. Since stopping this drug all her symptoms resolved, and she subsequently had no evidence of cardiac disease.

\section{Case Report}

A 61-year-cld housewife was admitted on 29 December 1972 with a 24-hour history of palpitations and acute dyspnoea. She had previously been in good health, but four months earlier she had become depressed and was treated with imipramine $25 \mathrm{mg}$ three times a day. During this period she had experienced occasional short-lived attacks of palpitations but had no other cardiac or systemic symptoms. In 1960 she had been found to be hypothyroid and had since taken thyroxine $0.3 \mathrm{mg} /$ day.

On admission she had the clinical and radiological features of acute pulmonary oedema. An E.C.G. showed rapid atrial fibrillation and left bundle-branch block, and in the absence of any other specific features a provisional diagnosis of acute myocarditis was made. She 